Irish Math. Soc. Bulletin

Number 83, Summer 2019, 3-4

ISSN 0791-5578

\title{
Real Life Decisions
}

\author{
BERNARD BEAUZAMY
}

\begin{abstract}
We show that everyday decisions, which should be taken on rational grounds, are most of the time taken on irrational basis, with very little room for mathematics. The reason is that data are not sufficient in order to ensure a proper scientific treatment of the problem, which is, therefore, treated in a completely nonscientific way. The mathematical community should develop tools for decision help in such situations.
\end{abstract}

1. All of us have to take decisions, on an everyday basis, and we all wish these decisions to be taken on rational grounds, perhaps even on scientific grounds: that would be great! Of course, we all know that this is not possible in general, since most decisions are taken on moral, religious, or emotional basis. Still, we have the impression that, in a few cases, Reason might enter. For instance, the width of a road (one lane, two lanes or more) might be decided from traffic data.

The academic community, in mathematics, has a strong feeling, even a conviction: they are completely certain that such problems should be addressed with the use of mathematics. However, this is not the case in practice, as we will see: even rational problems, such as the width of the road, are solved by irrational means, containing no mathematics. The reason is that, for the mathematical tools to apply, a lot of data would be needed: precise information about traffic at various times, the nature of the environment (characteristics of the landscape), pluviometry, and so on. Since these data are incomplete or missing, the decision will be taken based on rough information. Of course, if the land nearby belongs to a guy the Mayor does not like, the road will be promptly enlarged. Conversely, if a protected animal was seen nearby, the road will never be enlarged. Otherwise, it depends on budgets, public demand, severity of past accidents and many other factors, as usual in a modern democracy, with very little room for mathematics.

The result of such an attitude, for the academic community in mathematics, is quite negative: they have lost a lot of teaching duties, which are now taken by Engineering departments, Environment departments, and so on: since they could not provide a satisfactory answer, the mathematicians left the decision to people who come with "rough" tools, more appropriate in such situations.

Let us look more in detail at a specific example, which seems completely rational, namely predictive maintenance. An industry has a set of equipment (say for instance electricity distribution, and the equipment would be transformers), and wonders about the replacement of this equipment. All readers with good faith will admit that this problem has no moral contents. Some, with ecological minds, might consider that it should be addressed on a religious side: since the French transformers carry nuclear electricity, they should be destroyed, not replaced. Let us skip this debate.

2010 Mathematics Subject Classification. 00-01, 00A09.

Key words and phrases. Decision help, precision of the data.

Received on 16-11-2018; revised 19-5-2019. 


\section{The collapse of Operation Research}

Any mathematician from the academic world will consider that the above problem should be addressed by means of Operation Research Theory, which will provide a complete answer: not only which transformers and when, but also location of parts, size of teams, timetables for their actions, minimal budget and so on. But this is not so in practice: all necessary data are missing. One does not know much about the transformers (date of installation, dates of previous repairs), even less about the parts which might be necessary (are the old ones still produced?) and almost nothing about the people who are in charge. And about the budget, the question is: this year, or next decade?

Therefore, no Electricity Company solves this problem by means of Operation Research. What they do is this: they wait until something happens (usually after a storm), and then they fix as quickly as they can.

\section{What the problem is}

If we look more deeply at the problem, we see indeed that it is not simple at all. Roughly speaking, we have the choice between three possible attitudes:

(1) We double each item of equipment, so that there is a constant supply of electricity. This is very costly, and done only in critical situations, where no interruption is allowed (hospitals, for instance).

(2) We keep a lot of parts in some central place, and if some failure occurs, we take the part where it is and bring it quickly to the transformer to be repaired. This solution is rather cheap, but still some interruption will occur in case of a failure.

(3) We repair and replace the transformers in advance, according to some predefined scheme.

\section{Approaching a solution}

From a mathematical point of view, the third approach should have our preference. Each year, according to the existing budget, we can define how many transformers should be addressed. Then, a simple statistical survey will allow us to understand the structure of the availability. For instance, the transformers should last at least 50 years, but if they are close to the sea, they live much less. So, we will draw up very rough probability laws, saying: for this type, at this place, life expectancy is more than 40 years, and for this type at that place, less than 30 years.

Still, this is not a complete, solid, basis for decision, since some transformers are more critical than others. So one has to find, among the most critical, the ones which are oldest or more exposed.

One sees, from this example, that the decision will be taken on grounds which are not mathematical, but still contain a significant amount of mathematics, which is good for us. In any case, one should always remember that, as we say in French: Satan conduit le bal.

Bernard Beauzamy, born 1949, was University Professor 1979-1995. In 1995, he created the Company "Société de Calcul Mathmatique SA" and has been Chairman and Chief Executive Officer of this Company since then.

PDG, Société de Calcul Mathématique SA, 111 Faubourg Saint Honoré, 75008 Paris, FRANCE.

E-mail address: bernard.beauzamy@scmsa.com 